\title{
A MULTI-STAGE NEURAL NETWORK CLASSIFIER FOR ECG EVENTS
}

\author{
H. Gholam Hosseini ${ }^{1}$, K. J. Reynolds ${ }^{2}$, D. Powers ${ }^{2}$ \\ ${ }^{1}$ Department of Electrotechnology, Auckland University of Technology, Auckland, New Zealand \\ ${ }^{2}$ School of Informatics and Engineering, Flinders University, Adelaide, Australia
}

\begin{abstract}
In this paper, a multi-stage network including two multilayer perceptron (MLP) and one self organizing map (SOM) networks is presented. The input of the network is a combination of independent features and the compressed ElectroCardioGram (ECG) data. The proposed network as a form of data fusion, performs better than using the raw data or individual features. We classified six common ECG waveforms using ten ECG records of the MIT/BIH arrhythmia database. An average recognition rate of 0.883 was achieved within a short training and testing time.
\end{abstract}

Keywords - ECG event classifier, artificial neural network (ANN), ECG signal diagnosis

\section{INTRODUCTION}

Arrhythmias appear as deformation of the ECG waveform and can be subdivided into two groups; (i) events: which are single ectopic occurrences and, (ii) patterns: which are a continuous series of beats or arrhythmia [1].

Computer-aided diagnosis of cardiac events assists specialists to determine the appropriateness and nature of medical intervention. This is an active area of research and needs an in depth study into the classification of the ECG signals into different classes based upon the heart abnormalities.

Recent developments in computer-aided diagnosis of cardiac events have been successfully applied to the problem of automatic ECG classification. A number of algorithms have been developed in enhancing intelligent classification of the ECG signals [2-5]. Among them, the ANN-based ECG waveform discrimination algorithms, with classification rates comparable to those of the expert cardiologists, have been widely adopted by ECG signal professionals [6-8,3].

Examples of ANN based classifiers as applied to the ECG signals are the supervised back propagation (BP) network and the unsupervised Kohonen feature maps (KFM) network [9].

The results of an investigation using ANNs for ECG feature recognition are presented in Zhu's work [6]. ANNs are also suitable for patient-adaptable heart beat classifiers with the advantage of requiring no knowledge of the input distribution. A novel ECG monitoring system utilising two BP networks has been developed and achieved an acceptable performance after a short training procedure [7].

In other research, an ANN approach in which all parts of each heart beat play their role in the classification, has been proposed [8]. Short processing time and insensitivity to noise are the main reported achievements of this method.

An application of neural networks in classification of cardiac arrhythmias using fuzzy ART-MAP (adaptive resonance theory) has been investigated [9]. This relatively simple architecture with an easy and fast-to-train network was able to perform efficient classification of normal and PVC beats.
In another approach to the design of patient-adaptive classifiers, an ANN related algorithm was proposed based on the self-organization map (SOM), linear vector quantisation (LVQ) along with the mixture of experts (MOE) method [4]. SOM and LVQ together were used to train the patientspecific classifier, and MOE was used to facilitate the combination of the original and patient-specific classifiers to realise patient-adaptation. The original classifier used a large ECG database and the patient-specific classifier was trained specifically with the patient ECG data.

More recently, an ECG classifier using a modified decision-based ANN has been designed [10]. This classifier was able to diagnose the cardiac disease based on the knowledge acquired from the training. Therefore, the size of this network varied as the training procedure progressed.

There are several shortcomings with the above mentioned cardiac arrhythmia classifiers. For example, some techniques suffer from long processing times, other classifiers may depend on the patient ECG waveform characteristics and a few of the classifiers require noise and artifact removal. Moreover, many of the proposed algorithms analyse only one part of the signal and hence detect only one or two abnormalities [11]. It is observed that there exist more than 40 symbols in the MIT/BIH ECG database [12] to describe the number of different waveforms. Current classifiers have failed in clustering all abnormalities.

The other common problem of ECG signal classifiers is that structural complexity grows as the size of the training parameters increases. Moreover, performance of the classifiers is poor in recognising a specific type of ECG, which occurs rarely in a certain patient's ECG record.

This research is aimed to design the network architecture for detecting and classifying up to six different ECG waveforms including, normal $(\mathrm{N})$, premature ventricular contraction (PVC), paced beats $(\mathrm{P})$, right bundle branch block $(\mathrm{R})$, atrial premature beat $(\mathrm{A})$, and fusion of paced and normal beats (f). In addition, we aimed at decreasing the structural complexity of the classifier network.

\section{MethODOLOGY}

A set of least object-sensitive ECG features was selected to form the first part of the network input vector. The compressed ECG waveform [13] was used as the second part of the input vector. This strategy aimed to reduce the complexity of the network and facilitate the training phases of the network by employing a smaller training data size.

A preliminary multi-layer perceptron (MLP) classifier was designed to separate three most common ECG waveforms in the MIT/BIH database. The selected categories were N, PVC, and P beats. The second stage, with a similar architecture to the first, was designed to perform a supervised classification on the remaining waveforms. The selected 
waveforms for training and testing this MLP network were R and $\mathrm{A}$ beats.

Adding more stages to the output of the second stage can extend the clustering ability of the classifier. An unsupervised SOM was employed in the third stage of the network to perform further detection and classification.

"Fig. 1," depicts the flow diagram of the proposed multistage ECG events classifier. To reduce the classification error, a local test (LT) unit was designed to check each individual class and to test the output of the MLP network. As indicated in "Fig. 1", three test units named $\mathrm{LT}_{\mathrm{N}}, \mathrm{LT}_{\mathrm{V}}$, and $\mathrm{LT}_{\mathrm{P}}$ performed local tests on each heart beat and decided whether to keep or reject it. The rejected beats were considered as error and were classified into an unknown class $G_{x}$ for further classifications at the second stage of the classifier.

At the second stage two other abnormalities were detected and the remaining waveforms were considered as an unknown class $\mathrm{G}_{\mathrm{y}}$.

An unsupervised classifier such as SOM was added to the classifier for identifying those ECGs which were left by the second MLP as unrecognised group $\mathrm{G}_{\mathrm{y}}$.

The selected features of the ECG signal which form the first part of the classifier input vector were: the ST-segment area, ST-level, ST-slope, the R-S interval, the P-R interval, the QRS area, Q-T interval, R-wave amplitude, the QRS energy, auto-correlation coefficient, mean or expectation vector, and maximum amplitude of the signal histogram.

A fixed 52 sample interval centred at the QRS complex was selected with compression ratio $\mathrm{CR}=4$ for the compressed part of the input vector.



Fig. 1. Flow diagram of the multi-stage neural network classifier.
The extracted features plus 13 compressed ECG samples formed a 25-input network. The number of hidden layer neurodes was chosen as four, which is small enough for fast training and avoidance of overtraining, yet sufficiently large to give adequate network accuracy [14]. To achieve clustering of the ECG waveforms into four different classes, the number of network outputs was selected equal to two in each of the first and second stages. The proposed MLP with a single hidden layer is shown in "Fig. 2".

A target vector was arranged as the desired output for each class. Accompanying each record in the MIT/BIH database is an annotation file in which each heart beat has been identified by expert cardiologist annotators. This annotated information can be employed for designing the target vector and evaluating the classifier performance.

The clustering of the input data into four different classes by the first stage classifier was subject to some inaccuracies inherent in the pre-processing algorithms. QRS complex detection is a crucial task in ECG diagnosis and usually some errors are involved in these techniques which affect the feature extraction results. Moreover, the compressed signal as the second part of the classifier input vector generates some error.

The experimental results of both MLP and SOM architectures are presented in the following section.

\section{RESULTS}

A test set membership was determined arbitrarily after all details of the algorithms and parameters had been finalised. The test data sets contain a wide range of ECG waveforms including a total of 2032 normal, 364 PVC, 357 P, 363 R, 108 $A$, and 139 beats including $J$ and $F$ waveforms for the $G_{y}$ training set.

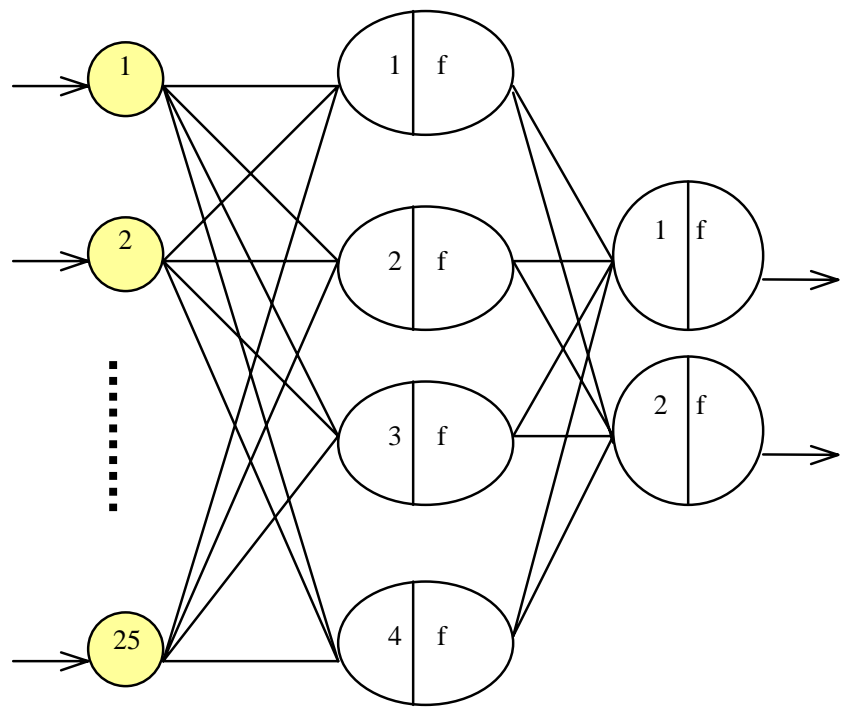

INPUT LAYER

HIDDEN LAYER

OUTPUT LAYER

Fig. 2. The proposed two-layered feed-forward MLP artificial neural network. 
The recognition rate is a statistical parameter used in estimating the performance of the classifiers. This factor is equal to one when all real events in the tested data are detected correctly. In the case of missing some true events during classification the recognition rate would fall below one. The corresponding recognition rates for ECG waveforms are assigned in Table I.

The results of applying statistical analysis to the classified ECG waveforms by the two-stage MLP network are summarised in Table II.

This classifier was not designed to detect other abnormalities and showed poor performance in recognising those beats. Therefore, group $\mathrm{G}_{\mathrm{y}}$ contained all unclassified beats and required further analysis by the next stage.

The designed SOM classifier is an unsupervised network and can separate different abnormalities in group $G_{y}$. This algorithm leads to placement of a set of prototype vectors in $\mathrm{G}_{\mathrm{y}}$, each of which corresponds to the best matching units in the two dimensional map for each data sample. Prototype vectors corresponding to nearby points on the feature map have nearby locations in input space [15]. This leads to the classification of different heartbeats among all unclassified beats in $\mathrm{G}_{\mathrm{y}}$.

Adding this stage to the proposed classifier improved the performance of the network in classifying additional ECG waveforms. The unsupervised SOM network in the final stage was able to cluster those unclassified beats as the results of its previous stage. For example, the fusion of paced and normal beats (f) is one of the abnormalities that were not detected correctly by the MLP stages. The SOM detected and clustered this group of ECG waveforms. However, due to the unsupervised characteristics of this network the output of this stage needed more attention for annotation purposes.

The SOM stage clustered the unclassified ECG waveforms without assigning any annotations to these groups.

TABLE I

THE RECOGNITION RATE AS ASSIGNED TO THE RELEVANT ECG WAVEFORMS

\begin{tabular}{ll} 
Recognition rate & ECG Waveform \\
\hline $\operatorname{Rr}_{N}$ & Normal (N) \\
$\operatorname{Rr}_{V}$ & PVC \\
$\operatorname{Rr}_{P}$ & Paced (P) \\
$\operatorname{Rr}_{X}$ & Other abnormal ECGs in stage one \\
$\operatorname{Rr}_{R}$ & Right bundle branch block (R) \\
$\operatorname{Rr}_{x}$ & Atrial premature (A) \\
$\operatorname{Rr}_{Y}$ & Other abnormal ECGs in stage two \\
\hline
\end{tabular}

TABLE II

THE STATISTICAL RESULTS OF THE ECG CLASSIFICATION BY MLP

\begin{tabular}{llllllll} 
Record & $\mathrm{Rr}_{\mathrm{N}}$ & $\mathrm{Rr}_{\mathrm{V}}$ & $\mathrm{Rr}_{\mathrm{P}}$ & $\mathrm{Rr}_{\mathrm{X}}$ & $\mathrm{Rr}_{\mathrm{R}}$ & $\mathrm{Rr}_{\mathrm{A}}$ & $\mathrm{Rr}_{\mathrm{Y}}$ \\
\hline 100 & 0.89 & 0.1 & $\mathrm{NA}$ & 0.30 & $\mathrm{NA}$ & 0.30 & $\mathrm{NA}$ \\
104 & 0.94 & 0.17 & 0.91 & 0.95 & $\mathrm{NA}$ & $\mathrm{NA}$ & 0.75 \\
106 & 0.99 & 0.98 & $\mathrm{NA}$ & $\mathrm{NA}$ & $\mathrm{NA}$ & $\mathrm{NA}$ & $\mathrm{NA}$ \\
107 & $\mathrm{NA}$ & 0.99 & $\mathrm{NA}$ & $\mathrm{NA}$ & $\mathrm{NA}$ & $\mathrm{NA}$ & $\mathrm{NA}$ \\
118 & $\mathrm{NA}$ & $\mathrm{NA}$ & $\mathrm{NA}$ & 0.89 & 0.86 & 0.60 & $\mathrm{NA}$ \\
119 & 0.98 & 1 & NA & NA & NA & NA & NA \\
123 & 0.99 & 0.50 & NA & NA & NA & NA & NA \\
217 & NA & 0.94 & 0.99 & NA & NA & NA & NA \\
232 & NA & NA & NA & 0.56 & 0.79 & 0.41 & NA \\
243 & 0.96 & NA & NA & 0.81 & NA & NA & 0.13 \\
\hline
\end{tabular}

Construction of the input vector based on the extracted features and compressed information of the signal improves the performance of the classifiers. For each record (as listed in Table II), the average recognition rate of the waveforms appearing in the record was found. The overall average recognition rate can be defined as the average of the average recognition rate of each of the records.

An average recognition rate of 0.883 was achieved testing ten ECG records of the MIT/BIH arrhythmia database.

The first stage network was trained with two different sized training sets to find any improvement or deterioration of the network due to reducing the size of training set. The larger training set contains 528 trained exemplars, which are indicated in Table III, and the smaller one contains 368 trained exemplars listed in Table IV.

The results of training with the smaller and larger sizes are summarised in Table V.

As indicated in Table V, after 50,000 training epochs, a lower mean square error (MSE) and a smaller gradient were achieved using the smaller size of training set. The network performed better in training with the smaller size of training set but had poor performances when applied to the evaluation or test sets.

\section{DISCUSSION}

The problem of ECG variations among persons with the same cardiological conditions affects the performance of ANN-based cardiac arrhythmia classifiers. In this study, the least patient-dependent features were selected among hundreds of measurable features [16]. The features were measured from the QRS complex, the ST-segment, $\mathrm{P}$ wave, T wave, statistical parameters, and histogram of the signal to cover the important diagnostic information of cardiac events. The feature extraction criterion enhanced the reliability and decreased the structural complexity of the ECG classifier.

TABLE III

THE LARGER TRAINING SET FOR THE FIRST STAGE MLP

\begin{tabular}{lll}
$\begin{array}{l}\text { Class } \\
\text { name }\end{array}$ & $\begin{array}{l}\text { Class } \\
\text { Description }\end{array}$ & $\begin{array}{l}\text { Number of } \\
\text { trained exemplars }\end{array}$ \\
\hline $\mathrm{N}$ & Normal beats & 160 \\
$\mathrm{PVC}$ & Premature ventricular contraction & 80 \\
$\mathrm{P}$ & Paced beats & 224 \\
$\mathrm{Gx}$ & Any beats except N, PVC, and P & 64 \\
\hline
\end{tabular}

TABLE IV

THE SMALLER TRAINING SET FOR THE FIRST STAGE MLP

\begin{tabular}{lll}
$\begin{array}{l}\text { Class } \\
\text { name }\end{array}$ & $\begin{array}{l}\text { Class } \\
\text { Description }\end{array}$ & $\begin{array}{l}\text { Number of } \\
\text { trained exemplars }\end{array}$ \\
\hline $\mathrm{N}$ & Normal beats & 100 \\
$\mathrm{PVC}$ & Premature ventricular contraction & 80 \\
$\mathrm{P}$ & Paced beats & 124 \\
$\mathrm{Gx}$ & Any beats except N, PVC, and P & 64 \\
\hline
\end{tabular}

TABLE V

THE RESULTS OF TRAINING THE FIRST STAGE WITH TWO TRAINING SETS

\begin{tabular}{lll} 
Number of trained exemplar & MSE & Gradient \\
\hline 528 & 0.0404 & 0.0090 \\
368 & 0.0121 & 0.0034 \\
\hline
\end{tabular}


Also the compressed form of the waveform was used instead of the raw data as the second part of the input vector. This method improved the speed, noise immunity, and simplicity of the classifier.

Among different investigated network structures the proposed network shown in "Fig. 1", possessed the most appropriate structure for ECG event classification. A MLP network in which a prediction of the first order error is an input to the second level network was used to improve the performance of the multi-stage classifier when a particular feature does not work well. The first stage MLP classified the most common ECG waveforms in the tested database with an average recognition rate of 0.818 . The second stage MLP increased the overall recognition rate to 0.883 and detected two more abnormal waveforms. The second stage error, $G_{y}$, was considered an input to the SOM stage for performing unsupervised signal classification.

\section{CONCLUSION}

The results of applying the proposed multi-stage classifier to the tested records confirmed the objectives of this research, i.e.

- To improve the reliability of the heart beat classifiers,

- To increase the accuracy of diagnosis where the classifier deals with a large group of abnormal ECGs,

- To decrease the structural complexity of the classifier networks.

The design of a perceptron network is constrained completely by the problem to be solved. The number of network inputs and the number of neurodes in each layer were constrained by the number of inputs and outputs required by the problem.

Due to the selection optimum number of neurodes in the hidden layer and adequate size of training set, overtraining was not a problem in the scope of the proposed networks.

The MLP network as a form of data fusion, employs a combination of independent features and the compressed ECG data and performs better than using the individual features. The idea of using a multi-stage network in this study is particularly appropriate for signals with a chaotic/fractal nature. Due to the unsupervised characteristics of SOM network the clustering results of this stage should be annotated by the cardiologists.

An average recognition rate of 0.883 was achieved combining multi-stage networks and performing local tests on the detected waveforms.

\section{REFERENCES}

[1] A. Rasiah and Y. Attikiouzel, "A syntactic approach to the recognition of common cardiac arrhythmias within a single ambulatory ECG trace," The Australian Computer journal, vol. 26, pp. 102-112, August 1994.

[2] V. X. Afonso and W. J. Tompkins, "Detection ventricular fibrillation: Selecting the appropriate time-frequency analysis tool for the application," IEEE Engineering in Medicine and Biology, pp. 152-159, March/April 1995.

[3] B. Celler and P. Chazal, "Low computational cost classifiers for ECG diagnosis using neural networks," in Proceedings of 20th Annual International Conference of
IEEE/EMBS, vol. 20, (Hong Kong), pp. 1337-1340, 20th Annual Int. Conf. IEEE Engineering in Medicine and Biology Society, Oct 29-Nov 11998.

[4] Y. H, Hu, S. Palreddy, and W. J. Tompkins, "A patient adaptable ECG beat classifier using a mixture of experts approach," IEEE Trans. Biomed. Eng., vol. 44, Pp. 891-900, September 1997.

[5] Y. S. Tsai, B. N. Hung, and S. F. Tung, "An experiment on ECG classification using back-propagation neural network," in Proceedings of Annual International Conference of IEEE/EMBS, pp. 1463-1464, Annu. Int. Conf. IEEE Engineering Medicine and Biology Society, 1990.

[61 K. Zhu, ECG feature recognition using artificial neural networks. PhD thesis, Uni. of Essex (U.K.), Dissertation abstracts international 55-01C, 1992.

[7] R. Watrous and G. Towell, "A patient-adaptive neural network ECG patient monitoring algorithm," Computer in cardiology, 1995.

[8] A. Badr, A computer environment for ECG waveforms morphological classification. $\mathrm{PhD}$ thesis, Rijksuniversiteit Te Gent (Belgium), 1993.

[9] S. Han, classification of cardiac arrhythmias using Fuzzy ARTMAP. PhD thesis, Florida Inst. of Tech. Melbourne, Florida, 1993.

[10] B. P. Simon and C. Eswaran, "An ECG classifier designed using modified decision based neural networks," Computer and Biomedical Research, no. 30, pp. 257-272, 1997.

[11] A. S. Miller, B. H. Blott, and T. K. Hames, "Review of neural network applications in medical imaging and signal processing," Medical and Biological Engineering and Computing, pp. 449-464, September 1992.

[12] G. Moody and R. Mark, MIT-BIH Arrhythmia Database Directory. MIT-BIH Database Distribution, Massachusetts Inst. Technol., 1992.

[13] H. Gholam Hosseini, H. Nazeran, and B. Moran, "ECG compression: Evaluation of FFT, DCT, and WT performance," Australian Physical and Engineering Science in Medicine, vol. 21, no. 4, pp. 186-192, 1998.

[14] C. Looney, Pattern recognition Using Neural Networks. http://www.oup-usa.org: Oxford University Press, 1997.

[15] C. M. Bishop, Neural Networks for Pattern Recognition. Claredon Press. Oxford, 1995.

[16] P. Chazal and B. Celler, "Selecting a neural network structure for ECG diagnosis," in Proceedings of 20th Annual International Conference of IEEE/EMBS, vol. 20, (Hong Kong), pp. 1422-1425, 20th Annual Int. Conf. IEEE Engineering in Medicine and Biology Society, Oct 29-Nov 1 1998. 